\title{
Neurology - to be or not to be of the cannabis-based medicine?
}

\author{
Natalia Szejko \\ Department of Neurology and Bioethics, Medical University of Warsaw, Poland
}

This edition of the Polish Journal of Neurology and Neurosurgery contains a Leading Topic dedicated to the use of cannabis-based medicine (CBM) in neurology. Growing evidence supports the application of CBM in a variety of neurological disorders such as epilepsy [1-5], multiple sclerosis (MS) [6-9], chronic pain [10], agitation in Alzheimer's Disease [11], Parkinson's Disease (PD) [12-14], Huntington's Disease [15, 16], Gilles de la Tourette Syndrome (GTS) [17], and other movement disorders [15]. Although there are disparities regarding the level of evidence for the use of CBM in diverse neurological disorders, with the highest being in epilepsy and MS, in all cases it is still considered an adjuvant and/or experimental therapy. In other fields of medicine such as pain medicine [18] or palliative care [19], CBM has already been recognised in official guidelines and this is one reason why this field of study is gaining more traction. In everyday practice, we encounter patients showing increased interest concerning CBM. Therefore, physicians are being challenged to establish their own point of view, which has to be based on current evidence rather than case reports or internet advice.

The Leading Topic comprises four articles that tackle different aspects of CBM use and offer a wide overview, from general information to detailed aspects concerning a particular disease. An extensive overview of the therapeutic potential and pharmacology of cannabinoids is presented by Śmiarowska, Białecka and Machoy-Mokrzyńska [20]. The authors discuss the nomenclature used to describe CBM and the pathophysiological background behind the function of the endocannabinoid system (ECS), and the distribution and function of cannabinoid receptor type 1 (CB1) and cannabinoid receptor type 2 (CB2), the main endocannabinoid receptors. Finally, the authors discuss the available evidence regarding the therapeutic application of CBM in a variety of indications, not only in neurology, and the potential neuroprotective effect of CBM and the hazards related to the use of cannabinoids.
Along with this general article, three more included in this Leading Topic are dedicated to the use of CBM in neurological disorders such as epilepsy. Mazurkiewicz-Bełdzińska and Zawadzka [21] write about the use of cannabidiol (CBD) in the treatment of epilepsy, as this is the only CBM that has been proved to be effective in a number of clinical trials and has received approval both from the American Food and Drug Agency (FDA) and the European Medicines Agency (EMA) for this indication, especially in syndromes known for their high refractoriness, such as Lennox-Gastaut Syndrome [22], Dravet Syndrome [23] and tuberous sclerosis [24]. The authors emphasise the theoretical background to CBM use in epilepsy, and give an overview of the available randomised controlled trials (RCT) and open trials.

Another article in this Leading Topic by Figura, Koziorowski and Sławek [13] presents the results of their systematic review of the use of CBM in PD. Following the usually applied methodological approach for meta-analysis, the authors were able to identify 569 papers regarding CBM in PD, but only seven of these were RCTs. Although analysis of cumulative data coming from these trials did not confirm CBM's effectiveness for the treatment of motor symptoms in PD, the authors concluded that non-motor symptoms such as pain, sleeping problems, and anxiety respond better to CBM than do motor symptoms, and therefore should be further investigated.

The last article in this Leading Topic, by Szejko et al. [17], is dedicated to the use of CBM in GTS. Although two small RCTs $[25,26]$ have demonstrated that CBM could be successfully used for the treatment of tics, both the newest Guidelines of the European Society for the Study of Tourette Syndrome $[27,28]$ as well as those of the American Academy of Neurology [29] recommend the use of CBM only in refractory, treatment-resistant cases. CBM therapy for this indication is still considered experimental. Recently, the largest RCT to test the efficacy and safety of nabiximols in GTS,

Address for correspondence: Natalia Szejko, MD, PhD, Department of Neurology and Bioethics, Medical University of Warsaw, Litewska 14 a Str., 00-581 Warsaw, Poland; e-mail: natalia.szejko@wum.edu.pl

Received: 27.01.2022 Accepted: 27.01.2022

This article is available in open access under Creative Common Attribution-Non-Commercial-No Derivatives 4.0 International (CC BY-NC-ND 4.0) license, allowing to download articles and share them with others as long as they credit the authors and the publisher, but without permission to change them in any way or use them commercially. 
CANNA-TICS [30], has been completed. The results of this trial will further elucidate the scientific rationale behind the use of CBM in GTS.

To sum up, CBM seems to be an important therapeutic avenue, especially in treatment-resistant cases of some neurological conditions, but it is still considered an experimental therapy. While reviewing the available evidence, it is clear that the majority of papers dedicated to the topic of CBM in neurology are case reports, case series and open studies with no randomisation. We still lack data with the highest level of evidence (RCTs). While an increasing amount of evidence explains the pathophysiological rationale behind CBM application in neurology, in many cases it is still based on merely theoretical or animal models. It also seems that diverse compounds (e.g. pure tetrahydrocannabinol (THC), CBD, nabiximols and medicinal cannabis) are more effective in different indications.

For example, $\mathrm{CBD}$ is more recommended in epilepsy, while THC, but not CBD, is more effective in GTS. However, in many diseases we still lack a direct comparison between different medications and it is unclear whether pure THC or $\mathrm{CBD}$ is superior to a combination of both used in nabiximols or medicinal cannabis.

As for medicinal cannabis, the issue is even more complicated because, in spite of a rigorous and regulated production process, medicinal cannabis contains more than 400 substances [31] that could influence the final therapeutic effect in particular indications. In addition, the amount of THC in each cannabis strain varies significantly. The mode of administration (i.e. inhalation, oral, vaporisation) has an impact on the pharmacokinetics and pharmacodynamics. Furthermore, depending on geographical region and differing state and national laws, the availability of CBM modalities differs.

Finally, the long-term side effects of CBM use are unclear, especially in children. While it has been demonstrated that abuse of recreational cannabis in childhood increases the risk of psychosis in adulthood [32], it is not clear whether the same risk is related to controlled CBM use under the supervision of a medical professional. Almost all researchers conclude their articles on this topic with a statement concerning the urgent need for the preparation of larger, multi-national RCTs that would help us to reach better conclusions regarding CBM safety and efficacy in neurological disorders.

We expect that this Leading Topic will encourage scientists to conduct such studies, and are confident that this will be of great interest to the readers of the Polish Journal of Neurology and Neurosurgery.

\section{References}

1. de Carvalho Reis R, Almeida KJ, da Silva Lopes L, et al. Efficacy and adverse event profile of cannabidiol and medicinal cannabis for treatment-resistant epilepsy: Systematic review and meta- analysis. Epilepsy Behav. 2020; 102: 106635, doi: 10.1016/j.yebeh.2019.106635, indexed in Pubmed: 31731110.

2. Caraballo R, Reyes G, Demirdjian G, et al. Long-term use of cannabidiol-enriched medical cannabis in a prospective cohort of children with drug-resistant developmental and epileptic encephalopathy. Seizure. 2022; 95: 56-63, doi: 10.1016/j.seizure.2022.01.001, indexed in Pubmed: 34999381.

3. Lattanzi S, Brigo F, Trinka E, et al. Efficacy and Safety of Cannabidiol in Epilepsy: A Systematic Review and Meta-Analysis. Drugs. 2018; 78(17): 1791-1804, doi: 10.1007/s40265-018-0992-5, indexed in Pubmed: 30390221.

4. Laux LC, Bebin EM, Checketts D, et al. CBD EAP study group. Longterm safety and efficacy of cannabidiol in children and adults with treatment resistant Lennox-Gastaut syndrome or Dravet syndrome: Expanded access program results. Epilepsy Res. 2019; 154: 13-20, doi: 10.1016/j.eplepsyres.2019.03.015, indexed in Pubmed: 31022635.

5. Zhu Z, Mittal R, Walser SA, et al. Complementary and Alternative Medicine (CAM) use in Children with Epilepsy. J Child Neurol. 2022 [Epub ahead of print]: 8830738211069790, doi: 10.1177/08830738211069790, indexed in Pubmed: 35099320.

6. Whiting PF, Wolff RF, Deshpande S, et al. Cannabinoids for Medical Use: A Systematic Review and Meta-analysis. JAMA. 2015; 313(24): 2456-2473, doi: 10.1001/jama.2015.6358, indexed in Pubmed: 26103030.

7. Corey-Bloom J, Wolfson T, Gamst A, et al. Smoked cannabis for spasticity in multiple sclerosis: a randomized, placebo-controlled trial. CMAJ. 2012; 184(10): 1143-1150, doi: 10.1503/cmaj.110837, indexed in Pubmed: 22586334.

8. Prieto González JM, Vila Silván C. Safety and tolerability of nabiximols oromucosal spray: a review of more than 15 years" accumulated evidence from clinical trials. Expert Rev Neurother. 2021; 21(7): 755-778, doi: 10.1080/14737175.2021.1935879, indexed in Pubmed: 34092180.

9. Khan H, Ghori FK, Ghani U, et al. Cannabinoid and endocannabinoid system: a promising therapeutic intervention for multiple sclerosis. Mol Biol Rep. 2022 [Epub ahead of print], doi: 10.1007/s11033022-07223-5, indexed in Pubmed: 35182322.

10. Duarte RA, Dahmer S, Sanguinetti SY, et al. Medical Cannabis for Headache Pain: a Primer for Clinicians. Curr Pain Headache Rep. 2021; 25(10): 64, doi: 10.1007/s11916-021-00974-z, indexed in Pubmed: 34628531.

11. Russo EB. Cannabis Therapeutics and the Future of Neurology. Front Integr Neurosci. 2018; 12: 51, doi: 10.3389/fnint.2018.00051, indexed in Pubmed: 30405366.

12. Urbi B, Corbett J, Hughes I, et al. Effects of Cannabis in Parkinson's Disease: A Systematic Review and Meta-Analysis. J Parkinsons Dis. 2022; 12(2): 495-508, doi: 10.3233/JPD-212923, indexed in Pubmed: 34958046.

13. Figura M, Koziorowski D, Sławek J. Cannabis in Parkinson’s Disease - the patient's perspective versus clinical trials: a systematic literature review. Neurol Neurochir Pol. 2022 [Epub ahead of print], doi: 10.5603/PJNNS.a2022.0004, indexed in Pubmed: 34985112.

14. Peball M, Krismer F, Knaus HG, et al. Collaborators of the Parkinson's Disease Working Group Innsbruck. Non-Motor Symptoms in Parkinson's Disease are Reduced by Nabilone. Ann Neurol. 2020; 88(4): 712-722, doi: 10.1002/ana.25864, indexed in Pubmed: 32757413. 
15. Koppel BS, Brust JCM, Fife T, et al. Systematic review: efficacy and safety of medical marijuana in selected neurologic disorders: report of the Guideline Development Subcommittee of the American Academy of Neurology. Neurology. 2014; 82(17): 1556-1563, doi: 10.1212/ WNL.0000000000000363, indexed in Pubmed: 24778283.

16. Lim K, See YM, Lee J. A Systematic Review of the Effectiveness of Medical Cannabis for Psychiatric, Movement and Neurodegenerative Disorders. Clin Psychopharmacol Neurosci. 2017; 15(4): 301-312, doi: 10.9758/cpn.2017.15.4.301, indexed in Pubmed: 29073741.

17. Szejko N, Saramak K, Lombroso A, et al. Cannabis-based medicine in treatment of patients with Gilles de la Tourette syndrome. Neurol Neurochir Pol. 2021 [Epub ahead of print], doi: 10.5603/PJNNS. a2021.0081, indexed in Pubmed: 34708399.

18. Busse JW, Vankrunkelsven P, Zeng L, et al. Medical cannabis or cannabinoids for chronic pain: a clinical practice guideline. BMJ. 2021; 374: n2040, doi: 10.1136/bmj.n2040, indexed in Pubmed: 34497062.

19. MacDonald E, Farrah K. CADTH Rapid Response Reports. Medical Cannabis Use in Palliative Care: Review of Clinical Effectiveness and Guidelines - An Update. Ottawa (ON): Canadian Agency for Drugs and Technologies in Health. Copyright $\odot 2019$ Canadian Agency for Drugs and Technologies in Health. ; 2019.

20. Śmiarowska M, Białecka M, Machoy-Mokrzyńska A. Cannabis and cannabinoids: pharmacology and therapeutic potential. Neurol Neurochir Pol. 2022 [Epub ahead of print], doi: 10.5603/PJNNS. a2022.0015, indexed in Pubmed: 35133644.

21. Mazurkiewicz-Bełdzińska M, Zawadzka M. Use of cannabidiol in the treatment of epilepsy. Neurol Neurochir Pol. 2022 [Epub ahead of print], doi: 10.5603/PJNNS.a2022.0020, indexed in Pubmed: 35211946.

22. Devinsky O, Patel AD, Cross JH, et al. GWPCARE3 Study Group. Effect of Cannabidiol on Drop Seizures in the Lennox-Gastaut Syndrome. N Engl J Med. 2018; 378(20): 1888-1897, doi: 10.1056/ NEJMoa1714631, indexed in Pubmed: 29768152.

23. Devinsky 0, Cross JH, Wright S, et al. Cannabidiol in Dravet Syndrome Study Group. Trial of Cannabidiol for Drug-Resistant Seizures in the Dravet Syndrome. N Engl J Med. 2017; 376(21): 2011-2020, doi: 10.1056/NEJMoa1611618, indexed in Pubmed: 28538134.

24. Thiele EA, Bebin EM, Bhathal H, et al. GWPCARE6 Study Group. Add-on Cannabidiol Treatment for Drug-Resistant Seizures in Tuber- ous Sclerosis Complex: A Placebo-Controlled Randomized Clinical Trial. JAMA Neurol. 2021; 78(3): 285-292, doi: 10.1001/jamaneurol.2020.4607, indexed in Pubmed: 33346789.

25. Müller-Vahl KR, Schneider U, Koblenz A, et al. Treatment of Tourette's syndrome with Delta 9-tetrahydrocannabinol (THC): a randomized crossover trial. Pharmacopsychiatry. 2002; 35(2): 57-61, doi: 10.1055/s-2002-25028, indexed in Pubmed: 11951146.

26. Müller-Vahl KR, Schneider U, Prevedel H, et al. Delta 9-tetrahydrocannabinol (THC) is effective in the treatment of tics in Tourette syndrome: a 6-week randomized trial. J Clin Psychiatry. 2003; 64(4): 459-465, doi: 10.4088/jcp.v64n0417, indexed in Pubmed: 12716250.

27. Roessner V, Eichele H, Stern JS, et al. European clinical guidelines for Tourette syndrome and other tic disorders-version 2.0. Part III: pharmacological treatment. Eur Child Adolesc Psychiatry. 2021 [Epub ahead of print], doi: 10.1007/s00787-021-01899-z, indexed in Pubmed: 34757514.

28. Müller-Vahl KR, Szejko N, Verdellen C, et al. European clinical guidelines for Tourette syndrome and other tic disorders: summary statement. Eur Child Adolesc Psychiatry. 2021 [Epub ahead of print], doi: 10.1007/s00787-021-01832-4, indexed in Pubmed: 34244849.

29. Pringsheim T, Okun MS, Müller-Vahl K, et al. Practice guideline recommendations summary: Treatment of tics in people with Tourette syndrome and chronic tic disorders. Neurology. 2019; 92(19): 896-906, doi: 10.1212/WNL.0000000000007466, indexed in Pubmed: 31061208.

30. Jakubovski E, Pisarenko A, Fremer C, et al. The CANNA-TICS Study Protocol: A Randomized Multi-Center Double-Blind Placebo Controlled Trial to Demonstrate the Efficacy and Safety of Nabiximols in the Treatment of Adults With Chronic Tic Disorders. Front Psychiatry. 2020; 11: 575826, doi: 10.3389/fpsyt.2020.575826, indexed in Pubmed: 33324255.

31. Al Ubeed HM, Bhuyan DJ, Alsherbiny MA, et al. A Comprehensive Review on the Techniques for Extraction of Bioactive Compounds from Medicinal Cannabis. Molecules. 2022; 27(3), doi: 10.3390/ molecules27030604, indexed in Pubmed: 35163863.

32. Pardo M, Matalí JL, Sivoli J, et al. Early onset psychosis and cannabis use: Prevalence, clinical presentation and influence of daily use. Asian J Psychiatr. 2021; 62: 102714, doi: 10.1016/j.ajp.2021.102714, indexed in Pubmed: 34090251. 